\title{
A 20 Weeks Intact Missed Miscarriage in a Rudimentary Horn With latrogenic Perforation of the Unicornuate Uterus
}

\author{
Basel Khreisat ${ }^{\mathrm{a}, \mathrm{c}}$, Aiman Smadia , Fatima Al-Edwan ${ }^{\mathrm{a}}$, Ayyed Ashamaseen ${ }^{\mathrm{a}}$, \\ Hazem Mohammad Alkhaldi ${ }^{b}$, Yazid Jibrel ${ }^{\mathrm{a}}$
}

\begin{abstract}
Pregnancy in a rudimentary horn is a rare condition which carries a high risk for both, the mother and her fetus. We present a case of missed miscarriage at 20 weeks of gestation in rudimentary horn with iatrogenic perforation of the unicornuate uterus in a 26-yearold lady gravid 2 para 1 . Laparotomy was done, and excision of the rudimentary horn altogether with the dead fetus and repair of the perforated uterus was performed. It is the first time to be reported in Jordan.
\end{abstract}

Keywords: Miscarriage; Ectopic pregnancy; Rudimentary horn; Unicornuate uterus; Pregnancy

\section{Introduction}

The estimation of Mullerian duct malformation was found to be 1 in every 1,000 women $[1,2]$, and rudimentary horn with unicornuate uterus is one of that malformation. A rudimentary horn is found in $84 \%$ of unicornuate uteri $[2,3]$. Usually, non-communicating may consist of a functional cavity or a small solid muscle with no functional endometrium. Urinary tract anomalies coexistence is found in $36 \%$ of cases [4]. Rudimentary horn pregnancy usually ruptured in $90 \%$ of cases during the second trimester with fetal demise, and cases of pregnancy progressing to the third trimester resulted in a live birth after cesarean section has been documented [5].

\footnotetext{
Manuscript accepted for publication April 16, 2015

aDepartment of Obstetrics and Gynecology, King Hussain Medical Center, Amman, Jordan

bDepartment of Anesthesia, King Hussain Medical Center, Amman, Jordan, Amman, Jordan

${ }^{\mathrm{c} C o r r e s p o n d i n g ~ A u t h o r: ~ B a s e l ~ K h r e i s a t, ~ D e p a r t m e n t ~ o f ~ O b s t e t r i c s ~ a n d ~ G y n e-~}$ cology, King Hussain Medical Center, PO Box 71, 11133 Al Ameer Hamza Suburb, Amman, Jordan. Email: bkhreisat@yahoo.com
}

doi: http://dx.doi.org/10.14740/jmc2150w

\section{Case Report}

The 26-year-old lady, gravida 2, para 1, one male living, known to have hypothyroidism on L-thyroxine $100 \mathrm{mg}$ daily, presented to our outpatient clinic referred from district hospital after failure to terminate her missed miscarriage which was estimated to be 22 weeks of gestation. She was booked at that hospital with ultrasound scans which showed healthy pregnancy till the time of loosing fetal heart activity, she was unknown to have a unicornuate uterus although she underwent previously a c-section because of breech presentation, and it was not mentioned to be seen during the current pregnancy scans that she had any uterine abnormality. They used in total $20 \mathrm{~T}$-misoprostol 50 $\mu \mathrm{g}$ in addition to intra-uterine Foleys catheter. At presentation, she looked well, and her vital signs were stable, BP 115/75, PR 85 bpm, afebrile. She was admitted to the ward after being scanned and confirmed that she had missed miscarriage, and the measurement of fetal BPD and FL showed 19 weeks gestation. It was missed that she had a pregnancy in a rudimentary horn. Trial of termination using misoprostol $50 \mu \mathrm{g}$ in addition to intrauterine Foleys catheter was done, the other day, patient had mild lower abdominal pain, an ultrasound scan by a consultant raised the suspicion of extrauterine pregnancy with presence of some free fluid and clot free in the abdominal cavity, but the patient was still clinically stable with drop in her $\mathrm{Hb}$ by $1 \mathrm{~g} / \mathrm{dL}$, so laparotomy was decided upon her, as with stable clinical condition confirmatory MRI was done before surgery (Fig. 1,2). During laparotomy there was clot free blood in the abdominal cavity, intact pregnancy of rudimentary horn with perforated corneal side of unicornuate uterus resulting from the intrauterine catheter (Fig. 3), surrounded by hematoma at the site of perforation but no active bleeding. Evacuation of the clots and blood, incision was made in rudimentary horn and fetus was taken out (Fig. 4), then excision of rudimentary horn with its contents and finally suturing of the ruptured unicornuate uterus was performed, free drain was inserted. The patient received two units of packed RBCs and discharged home on the third day postoperatively in good condition.

\section{Discussion}

The incomplete development of one of the Mullerian ducts and the incomplete fusion with the contralateral side result in 


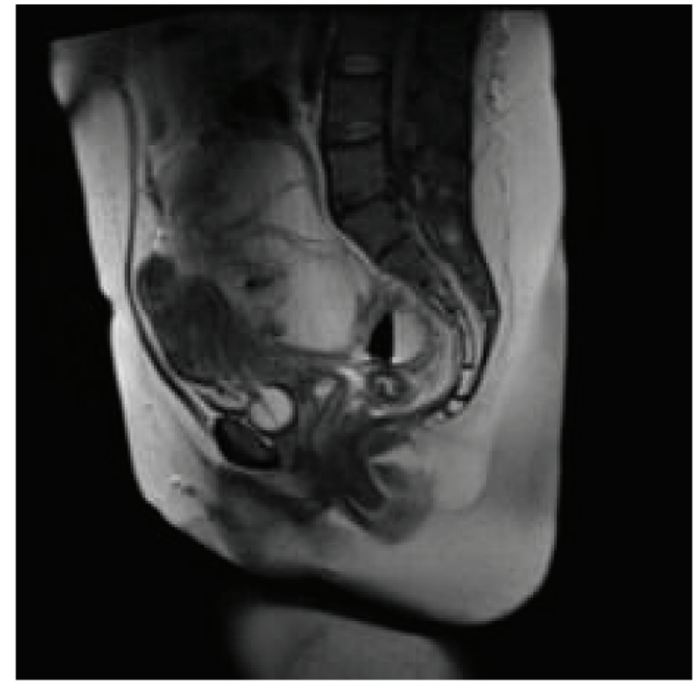

Figure 1. T2-wieghted sagittal MRI showing pregnancy in rudimentary horn, empty uterus with hematoma on the fundus.

the formation of a rudimentary horn with a unicornuate uterus. The estimated incidence of pregnancy in the rudimentary horn is estimated at 1 per 100,000 to 140,000 pregnancies $[6,7]$. Transperitoneal migration of the sperm or the fertilized ovum resulted in the pregnancy in a non-communicating rudimentary horn $[7,8]$.

Poor distensibility of the myometrium, dysfunctional endometrium and the variability in the thickness of the musculature of the rudimentary horn lead to rupture which is usually seen in the second trimester and can lead to life threatening condition for the mother resulting from hemoperitonium and shock [9]. Maternal mortality rate was reported to be $47.6 \%$ before 1900. Although rupture of the horn is still common, there is no reported maternal death since $1960[8,10]$.

Decreased blood supply and defective endometrium lead to missed miscarriage or intrauterine death in some cases [3], same as happened with our case.

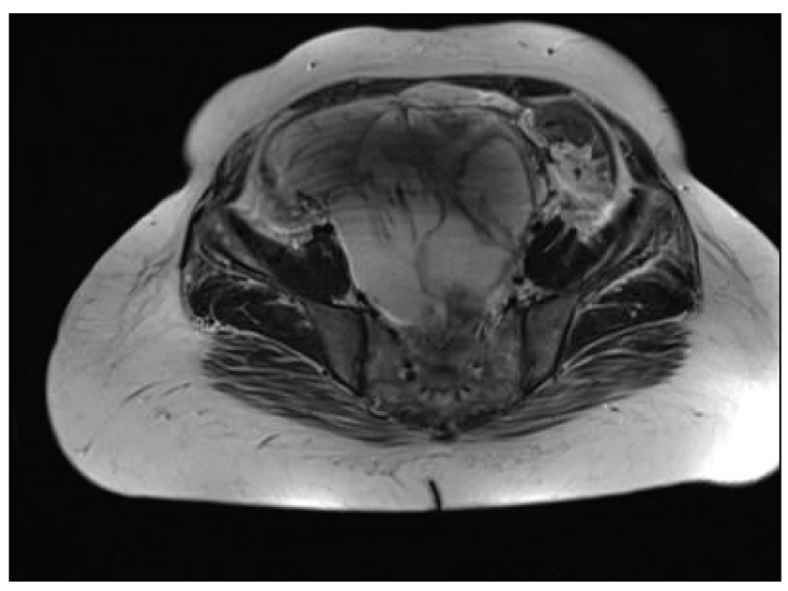

Figure 2. T2-wieghted horizontal MRI showing the empty unicornuate uterus with pregnancy outside in the rudimentary horn.

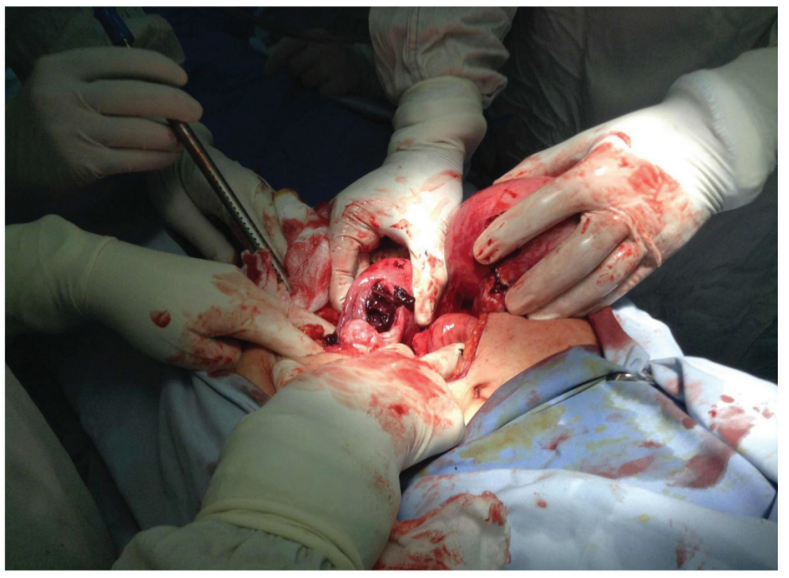

Figure 3. Perforated unicornuate horn with intact rudimentary horn.

The key to successful management of rudimentary horn pregnancy is the early diagnosis. The following criteria for ultrasonographic diagnosis were proposed by Tsafrir et al [11]: a pseudo pattern of an asymmetrical bicornuate uterus, absent visual continuity tissue surrounding the gestational sac and the uterine cervix, and the presence of myometrial tissue surrounding the gestational sac. The sensitivity of ultrasound is only $26 \%$ and decreases as the pregnancy advances [4] and can be missed in inexperienced hands as in our patient. An MRI examination has proven to be a useful tool for the diagnosis as well as for the choice of surgical approaches [12]. Unfortunately, our case was misdiagnosed initially as an intrauterine missed miscarriage, ultrasound by experienced consultant raised the suspicion of the diagnosis in addition to MRI, but definitive findings were revealed after exploration. Occasional cases (gynecologic and obstetric) that required further evalua-

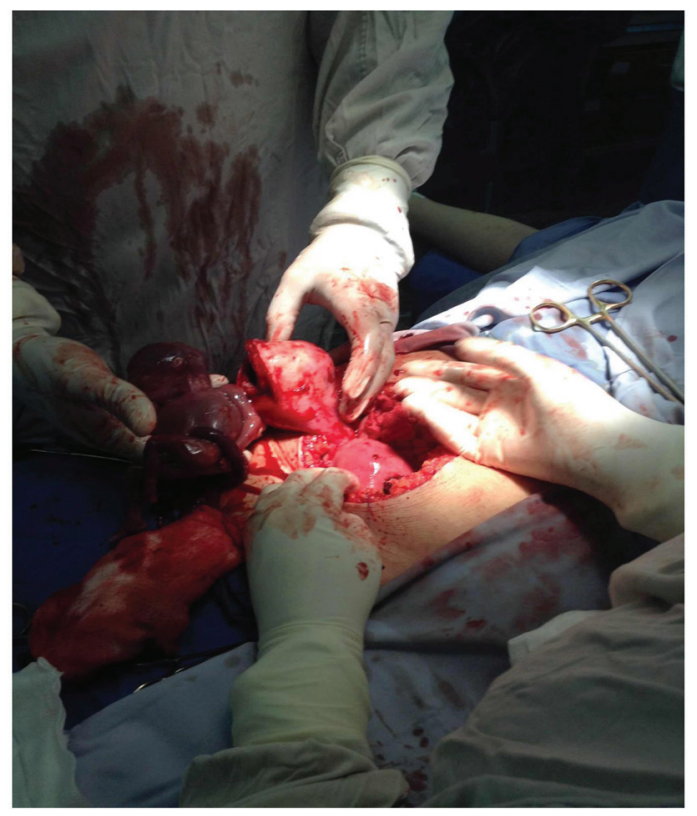

Figure 4. Dead fetus after incising the rudimentary horn. 
tion because of confusion in the diagnosis have been reported in the literature $[12,13]$. Intrauterine fetal demise was a diagnosis of rudimentary horn pregnancy reported by Buntungu et al where induction with misoprostol failed leading to the suspicion of ectopic pregnancy [14].

Surgical removal of rudimentary horn is the primary strategy of management [10]. Dicker et al made early diagnosis and removed a small rudimentary horn through the suprapubic laparoscopic port [15]. Successful medical management by methotrexate at an early gestation was reported by Edelman et al [16]. Rudimentary horn pregnancy should be considered as a differential diagnosis to bicornuate intrauterine pregnancy, and early detection and management should be done. Ultrasound examination should include renal system due to strong association between uterine anomalies with renal anomalies.

\section{Conflict of Interest}

Authors declare no conflict of interest.

\section{References}

1. Heinonen PK. Unicornuate uterus and rudimentary horn. Fertil Steril. 1997;68(2):224-230.

2. Nagarathna G, Mahesha Navada H, Poornima B, Bhat R. Pre-rupture diagnosis and management of rudimentary horn pregnancy in second trimester. Int $\mathrm{J}$ Pharm Biomed Res. 2011;2(3):179-181.

3. Heinonen PK. Clinical implications of the unicornuate uterus with rudimentary horn. Int J Gynaecol Obstet. 1983;21(2):145-150.

4. Jayasinghe Y, Rane A, Stalewski H, Grover S. The presentation and early diagnosis of the rudimentary uterine horn. Obstet Gynecol. 2005;105(6):1456-1467.

5. Shin JW, Kim HJ. Case of live birth in a non-communicating rudimentary horn pregnancy. J Obstet Gynaecol Res.
2005;31(4):329-331.

6. Sevtap HK, Aral AM, Sertac B. An early diagnosis and successful local medical treatment of a rudimentary uterine horn pregnancy: a case report. Arch Gynecol Obstet. 2007;275(4):297-298.

7. Kanagal DV, Hanumanalu LC. Ruptured rudimentary horn pregnancy at 25 weeks with previous vaginal delivery: a case report. Case Rep Obstet Gynecol. 2012;2012:985076.

8. Scholtz M. A full-time pregnancy in a rudimentary horn of the uterus. J Obstet Gynaecol Br Emp. 1951;58(2):293296.

9. Kriplani A, Relan S, Mittal S, Buckshee K. Pre-rupture diagnosis and management of rudimentary horn pregnancy in the first trimester. Eur J Obstet Gynecol Reprod Biol. 1995;58(2):203-205.

10. Nahum GG. Rudimentary uterine horn pregnancy. The 20th-century worldwide experience of 588 cases. J Reprod Med. 2002;47(2):151-163.

11. Tsafrir A, Rojansky N, Sela HY, Gomori JM, Nadjari M. Rudimentary horn pregnancy: first-trimester prerupture sonographic diagnosis and confirmation by magnetic resonance imaging. J Ultrasound Med. 2005;24(2):219-223.

12. Stitely ML, Hopkins K. Laparoscopic removal of a rudimentary uterine horn in a previously hysterectomized patient. JSLS. 2006;10(2):257-258.

13. Park JK, Dominguez CE. Combined medical and surgical management of rudimentary uterine horn pregnancy. JSLS. 2007;11(1):119-122.

14. Buntugu K, Ntumy M, Ameh E, Obed S. Rudimentary horn pregnancy: pre-rupture diagnosis and management. Ghana Med J. 2008;42(2):92-94.

15. Dicker D, Nitke S, Shoenfeld A, Fish B, Meizner I, BenRafael Z. Laparoscopic management of rudimentary horn pregnancy. Hum Reprod. 1998;13(9):2643-2644.

16. Edelman AB, Jensen JT, Lee DM, Nichols MD. Successful medical abortion of a pregnancy within a noncommunicating rudimentary uterine horn. Am J Obstet Gynecol. 2003;189(3):886-887. 